\title{
DigestJoin: Exploiting Fast Random Reads for Flash-based Joins
}

\author{
Yu Li, Sai Tung On, Jianliang Xu, Byron Choi, Haibo Hu \\ Department of Computer Science, Hong Kong Baptist University \\ Kowloon Tong, Hong Kong SAR, China \\ \{yli, ston, xujl, bchoi, haibo\}@comp.hkbu.edu.hk
}

\begin{abstract}
Flash disks have been an emerging secondary storage media. In particular, there have been portable devices, multimedia players and laptop computers that are configured with no magnetic disks but flash disks. It is envisioned that some RDBMSs will operate on flash disks in the near future. However, the I/O characteristics of flash disks are different from those of magnetic disks. Thus, in this paper, we study the core of query processing in RDBMSs - join processing - on flash disks. Specifically, we propose a new join method, called DigestJoin, to exploit fast random reads of flash disks. DigestJoin consists of two phases: (1) projecting the join attributes followed by a join on the projected attributes; and (2) fetching the full tuples that satisfy the join to produce the final join results. While the problem of tuple/page fetching with minimum $I / O$ cost (in the second phase) is intractable, we propose three heuristic fetching strategies. We have implemented DigestJoin on a real flash disk for performance evaluation. Experiments on TPC-H datasets show that DigestJoin clearly outperforms the traditional sort-merge join under various system configurations.
\end{abstract}

\section{INTRODUCTION}

Flash disks have been widely used in portable devices such as PDAs, smartphones and multimedia players, as well as in some laptop and desktop computers in the form of Solid State Drive (SSD). When compared to their magnetic counterpart, flash disks offer comparable storage space, better data access performance, better shock resistance, lower power consumption, lighter weight, smaller dimension and better noise resistance. Given such a wide range of advantages, flash disks have been a competitive candidate for the nextgeneration mass storage media.

Adopting flash disks as a secondary storage media, recent research (e.g., [11], [16], [17], [22]) has investigated the possibilities for developing flash-based RDBMSs. It has been remarked that flash disks have unique $\mathrm{I} / \mathrm{O}$ characteristics (see Table I for a comparison of I/O operation overhead between magnetic disks and flash disks). For instance, flash-based storage does not involve any mechanical components and hence there is a negligible seek time and rotational delay in reading or writing a page on a flash disk. While flash disks still have some overhead on each I/O operation, which is caused by the encapsulated logic for such purposes as wear leveling and internal caching [4], [6], such overhead can be more than 20 times smaller than its mechanical counterpart in magnetic disks. Recall that query processing algorithms on magnetic disks often spend an effort to avoid random I/O operations but exploit sequential I/O operations whenever possible. The
TABLE I

Magnetic Disk's Seeking vs. SSD's Overhead [12]

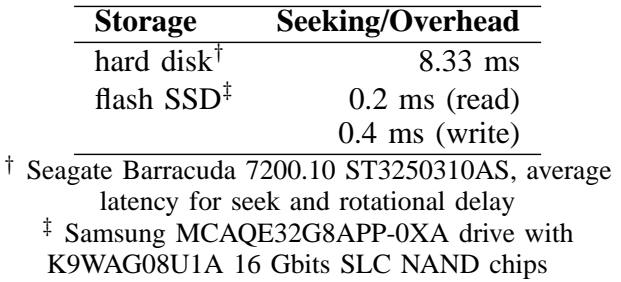

I/O characteristics of flash disks indicate that such an effort is no longer crucial in flash-based RDBMSs.

The state-of-the-art RDBMSs are mainly designed to operate on magnetic disks and therefore assume the I/O characteristics of magnetic disks. Among the operators of SQL (supported by all RDBMSs), joins are particularly I/O intensive and computationally expensive. Therefore, joins have been extensively studied in the literature [15]. In this paper, we focus on joins in the absence of indexes, i.e., non-index joins. There have been a few existing non-index join methods, e.g., nested-loop, sort-merge and hash joins. An objective is to reduce the $\mathrm{I} / \mathrm{O}$ opertions in performing the join.

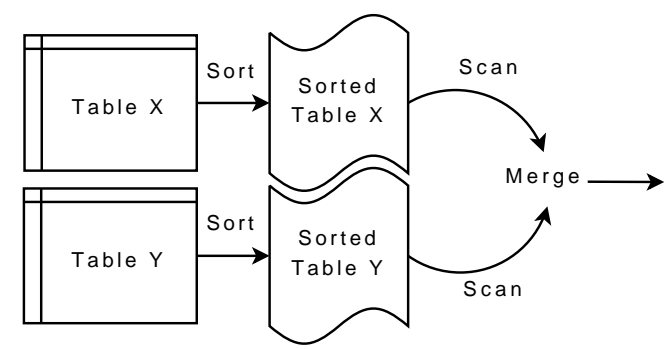

Fig. 1. Sort-Merge Join Algorithm

Consider the nested-loop join algorithm. Assume that the tables, that participate the join, do not fit into main memory. The nested loops encapsulate table scans which minimize the seek operations in performing the join. Similarly, consider the sort-merge join algorithm. It sorts the tables (using an external sort algorithm if necessary) by the join attributes. This facilitates table scans on the sorted tables in the merge phase of the algorithm, as illustrated in Fig. 1. This shows that in the absence of indexes, join algorithms have been minimizing the number of seek operations. Nevertheless, as discussed earlier, 
original tables according to tids. Fetching tuples from the original tables is usually performed at the granularity of pages in RDBMS. Thus, we have the second phase - page fetching. Although the page fetching will pay some cost of random reads, we hope that the cost is only a small part of the $\mathrm{I} / \mathrm{O}$ saving gained in performing digest table joining.

Page Fetching. A careful fetching schedule of tuples is critical to the page fetching problem. To illustrate that, we give a simple example. Suppose that we have the following digest join results: $\left(x_{1}, t i d_{x_{1}}, t i d_{y_{1}}\right),\left(x_{2}, t i d_{x_{2}}, t i d_{y_{2}}\right),\left(x_{3}, t i d_{x_{3}}, t i d_{y_{3}}\right)$ and $\left(x_{4}, t i d_{x_{4}}, t i d_{y_{4}}\right)$, and that tuples $t i d_{x_{1}}$ and $t i d_{x_{3}}$ are stored on page $A$, tuples $t i d_{x_{2}}$ and $t i d_{x_{4}}$ are on page $B$, tuples $t i d_{y_{1}}$ and $t i d_{y_{3}}$ are on page $C$, tuples $t i d_{y_{2}}$ and $t i d_{y_{4}}$ are on page $D$. If we have sufficient memory space, we may fetch all of these four pages, namely, $A, B, C$ and $D$, and keep them in the memory throughout the process of constructing the final join results. However, in practice, memory space is limited and, hence, we need to carefully schedule the page fetching to minimize the page read cost. Suppose that the memory space can hold two pages only. If we construct the final results in the order of $x_{1}, x_{2}, x_{3}$ and $x_{4}$, we need to fetch pages $A$ and $C$ for $x_{1}, B$ and $D$ for $x_{2}$, then $A$ and $C$ again for $x_{3}$, and finally $B$ and $D$ again for $x_{4}$. In this case, each page is fetched twice. Alternatively, we may swap the order of $x_{2}$ and $x_{3}$ in the schedule and then each page would be fetched only once. Therefore, as can be seen, the page fetching schedule has a great impact on the I/O cost. We will propose three strategies for page fetching in the next section.

Before we proceed to the next section, we present an example to exemplify how much DigestJoin can improve over a traditional join algorithm. Consider the following join on two TPC-H tables, where CUSTOMER and ORDERS are joined through the key C_CUSTKEY:

SELECT *

FROM CUSTOMER, ORDERS

WHERE CUSTOMER.C_CUSTKEY = ORDERS.C_CUSTKEY;

According to the schemas of TPC-H, the tuple size of a digest table is approximately $6 \%$ of that of the CUSTOMER table, and $9 \%$ of that of the ORDERS table. Assume that there are 10,000 and 5,000 pages for CUSTOMER and ORDERS, respectively. Consider the traditional sort-merge algorithm. Given a memory size of 20 pages, we need 4 and 3 passes to sort these two tables, respectively, and the total sorting cost is $4 \times 2 \times 10,000+$ $3 \times 2 \times 5,000=110,000 \mathrm{I} / \mathrm{Os}$. The next merge procedure will alternatively scan both of the sorted tables, leading to a total join cost of $110,000+10,000+5,000=125,000$ I/Os. Now if we employ DigestJoin, the digest tables extracted from CUSTOMER and ORDERS are of size 600 and 450 pages, respectively. Thus, the cost for building these two digest tables is $10,000+5,000+600+450=16,050 \mathrm{I} / O s$. Then, when applying the sort-merge join to the digest tables, both tables can be sorted in 3 passes, with a join cost of $3 \times 2 \times 600+$ $3 \times 2 \times 450+600+450=7,350 \mathrm{I} / \mathrm{Os}$. Hence, the total cost incurred in the first phase will be $16,050+7,350=23,400$
I/Os. Thus, as long as the second page fetching phase requires fewer than 101,600 I/Os, DigestJoin would outperform the traditional sort-merge join algorithm.

Discussions. The proposed DigestJoin method is appealing to flash-based storage media in two aspects. On one hand, it reduces the size of intermediate join results, thereby saving the I/O operations and particularly the temporary writes to flash disks during the join. This also implicitly leads to fewer erase operations on flash disks. On the other hand, DigestJoin is possible due to fast random reads of flash disks. In the second phase, the tuples involved in the join results are likely to be scattered over a flash disk. Thus, page fetching will incur quite a number of random read operations, which are extremely costly for magnetic disk-based storage media. For magnetic disks, the overhead of such random read operations may even exceed the I/O cost saved in the first phase. Flashbased storage media is particularly benefited from joining the digest tables, as random reads on flash disks are efficient.

\section{Page Fetching Strategies for DigestJoin}

An efficient page fetching strategy is important for $\mathrm{Di}$ gestJoin as it minimizes the number of page accesses when fetching the full tuples from the original tables to produce the final join results. In this section, we first formulate the page fetching problem as a graph problem and discuss its hardness. Then, we propose three heuristic-based strategies to address this problem.

\section{A. The Page Fetching Problem}

In DigestJoin, the outputs of the first phase are the digest join results. Based on that, in the second phase, disk pages containing the full tuples (or partial tuples containing required attributes in the projection list of the input query) are fetched to generate the final join results. The page fetching problem is to schedule a page fetching sequence to read all tuples in the join results with minimum number of page accesses.

To analyze the page fetching problem, we formulate it as a graph problem. A join graph is used to represent the relationship between the disk pages specified by the join results. The join graph is defined as an undirected bipartite graph $G=\left(V_{1} \cup V_{2}, E\right)$, where $V_{1}$ and $V_{2}$ denote the set of pages from the two original tables, respectively, and $E \subseteq V_{1} \times V_{2}$ denotes the set of page-pairs specified by the join results. Specifically, for each edge $\left(v_{a}, v_{b}\right) \in E$, there exists a tuple on page $v_{a}$ which joins with a tuple on page $v_{b}$. The join graph can be used to dynamically represent the remaining pages to be fetched and joined. An edge $\left(v_{a}, v_{b}\right)$ is removed from $E$ if pages $v_{a}$ and $v_{b}$ have been fetched into the main memory and the corresponding tuples on them have been joined. A vertex $v$ is removed from $\mathrm{G}$ once its out-degree becomes zero.

An example of a join graph is shown in Fig. 3, where vertices 1 and 2 represent the pages from one table, while vertices $a, b$ and $c$ represent the pages from the other table. An edge $(1, a)$ means there is/are tuple(s) on page 1 that can be joined with tuple(s) on page $a$. Similarly, edges $(1, b),(1, c)$, 
sequence which ensures each page is fetched at most once. This motivates us to propose the page-based fetching strategy.

Specifically, we build two kinds of temporary tables to assist page fetching. The first one is called fetching instruction table, which archives digest join results. After this table is filled with all digest join results, we sort its digest join results based on their page addresses. Thus, fetching tuples based on such sorted digest results avoids duplicated page fetching requests. However, tuples fetched according to their page addresses are generally not clustered on the join attributes. Hence, we have another temporary table called join candidate table to store the tuples fetched according to the fetching instruction table. Sort-merge join or hash join algorithm can then be applied on this table for producing the final join results.

To illustrate the page-based fetching strategy, let us recall the example on digest join results used in Section II. In practice, we may encode the tids in the form of "page_id:slot_number" when we generate the digest tables. Here, the results are $\left(x_{1}, \mathrm{~A}: 1, \mathrm{C}: 1\right)$, $\left(x_{2}, \mathrm{~B}: 1, \mathrm{D}: 1\right), \quad\left(x_{3}, \mathrm{~A}: 2, \mathrm{C}: 2\right) \quad$ and $\quad\left(x_{4}, \mathrm{~B}: 2, \mathrm{D}: 2\right)$. Two fetching instruction tables are then created: $f t_{1}=\{\mathrm{A}: 1, \mathrm{~B}: 1, \mathrm{~A}: 2, \mathrm{~B}: 2\}, f t_{2}=\{\mathrm{C}: 1, \mathrm{D}: 1, \mathrm{C}: 2, \mathrm{D}: 2\}$. We sort them according to page_id and obtain $f t_{1}=\{\mathrm{A}: 1, \mathrm{~A}: 2, \mathrm{~B}: 1, \mathrm{~B}: 2\}, f t_{2}=\{\mathrm{C}: 1, \mathrm{C}: 2, \mathrm{D}: 1, \mathrm{D}: 2\}$. The tuples now can be fetched efficiently. We then obtain two join candidates tables - jct $1=\left\{x_{1}, x_{2}, x_{3}, x_{4}\right\}$, $j c t_{2}=\left\{y_{1}, y_{2}, y_{3}, y_{4}\right\}$. By joining them again, we obtain the final join results.

The page-based strategy gives an efficient page fetching sequence with the cost of introducing some extra I/O cost for maintaining two temporary tables and another extra final join. We summarize the above discussions in Algorithm 2.

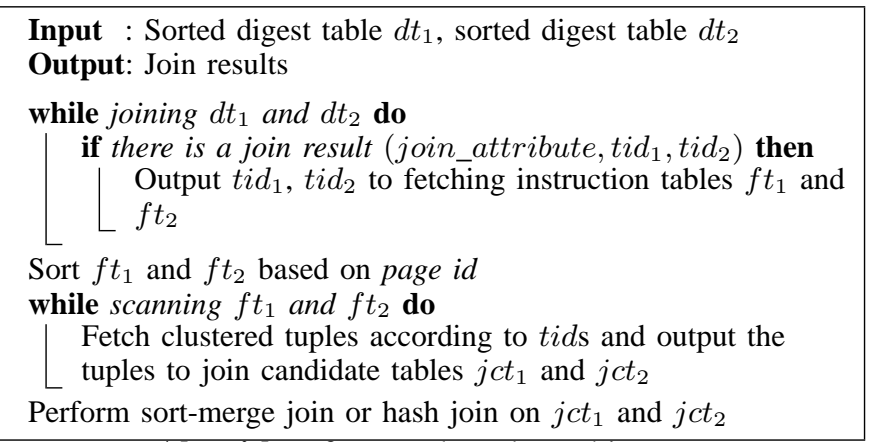

Algorithm 2: Page-based Fetching

\section{Graph-based Fetching Strategy}

Instead of archiving the digest join results in temporary tables, an alternative method is to archive it with the join graph. If the memory can hold all digest join results in the form of a join graph, we may find good heuristics to travel all edges to do the page fetching and joining near optimally. In the literature, two heuristics [3], [18] are in that direction, focusing on page fetching for index-based joins. The basic idea is to select a subgraph of a vertex which contains all its adjacent edges and requires the fewest non-resident pages to be fetched. Here, a non-resident page is a page that is not currently cached in the main memory. In other words, we check all subgraphs in the current join graph: 1) to identify whether each subgraph contains all edges of any vertex in the subgraph; and 2) to count how many vertices (i.e., pages) are not in the main memory. Then, the subgraph that satisfies the first condition and has the smallest value of the second quantity is selected. As iterating all subgraphs of the join graph makes the computational cost prohibitively high, an approximation method is to select the vertex with the fewest non-resident neighbors, together with all its neighbors in the join graph, called segment [3].

For example, let us reconsider the join graph in Fig. 3. The candidate segments are $\{1, a, b, c\},\{2, a, c\},\{a, 1,2\},\{b, 1\}$ and $\{c, 1,2\}$. If there has not been any page fetched into the main memory yet, we should select $\{b, 1\}$. This is because it has the fewest non-resident neighbors and implies the least page accesses. Let us assume that some pages have already been fetched into the main memory, say $c$. Then, we may select $\{2, a, c\}$ or $\{b, 1\}$. The reason is that both pages 2 and $b$ have only one non-resident neighbor, i.e., pages $a$ and 1 , respectively.

However, there are some challenges in applying this heuristic to our scenario. First, we observe that the devices installed with flash disks often have a limited memory space. In our research, we study joins on large tables that are larger than the size of available memory. Therefore, the memory space is not likely to hold the entire join graph for the analysis mentioned earlier. Furthermore, in order to avoid producing duplicate join results, we have to attach tids to the edges in the join graph in implementation. This is a rather subtle point. Consider the scenario where only page ids are attached to the join graph. Suppose we have an edge in the join graph indicating a join between page $a$ of table $X$ and page $b$ of table $Y$. When the memory space runs out, we conduct the join on pages $a$ and $b$ to reclaim space by removing the corresponding vertices and edges from the join graph. Next time, when another digest join result requiring page $a$ to join with page $b$ comes, duplicate join results will be generated. As there is no information on what results have already been produced, attaching tids to the edges can address this issue. However, it makes the size of the join graph grows faster and aggravates memory shortage.

Second, pre-allocating sufficient memory for caching pages for the join graph is not feasible in general. The heuristic we discussed above provides a rough bound on the maximum required size of the page cache, i.e., $\min \{\operatorname{sizeof}(X)$, sizeof $(Y)\}+1$, when joining tables $X$ and $Y$. However, if we could reserve so many pages for the page cache, we can simply load one table into the memory and use the remaining memory space to scan the other table to perform the join. Therefore, we need a careful implementation to effectively hold both the join graph and the page cache in a limited memory space.

In this subsection, we propose a graph-based fetching strategy which aims at achieving acceptable performance even when the memory available is highly limited. Fig. 5 gives an overview of the memory management for the graph-based 
We implemented DigestJoin with all three page fetching strategies in an experimental database system built on top of 16GB Mtron MSD-SATA3025 SSD. The experimental database system is designed to enable an easy evaluation on the performance of different join algorithms. It is composed of three components: raw storage manager, page-oriented buffer manager and query executor. The raw storage manager maintains a bunch of pre-allocated continuous space on the SSD and provides a page-oriented read/write interface. The other two components rely on this interface to perform I/O access to the SSD. The page-oriented buffer manager maintains a fixed number of memory pages, each of which has the same size as the ones in the raw storage manager. Any query processing in the query executor should interact with the memory pages in the buffer manager. The query executor provides facilities to implement and execute specific join algorithms. The system catalog maintains some statistics information, such as system parameters (e.g., page size, memory size, etc.), table summaries (e.g., \# tuples, \# pages, etc.), as well as join statistics (e.g., join selectivity).

Tables are organized on page-oriented space of the SSD. The data tuples are stored on the pages following a row-based storage scheme. Since we study non-index joins, we do not build any index for each table. To save space, we store data tuples in a variable-size format. Finally, the data tuples are imported into the storage in a random order. This is to simulate a general-case join where the join attribute values could be in any arbitrary order in the original tables.

The test dataset is taken from the TPC-H benchmark. In particular, we use one CUSTOMER table of TPC-H and perform a natural self-join through the key C_CUSTKEY. The selectivity is thus $100 \%$. In our implementation, we install a filtering function before we are about to get a join result. The filtering function will flip a coin to decide whether to drop the result or not. By controlling the flipping probability, we can simulate different degrees of selectivity. In particular, we can also control the selectivity on a page basis to simulate skewed join distributions.

We use the sort-merge join algorithm as a representative to evaluate the DigestJoin method, while expecting that similar performance results can be observed for nested-loop join. We have not compared the hash join since its performance depends on the quality of the hash function and more on the distribution of the join values. Specifically, the algorithms under evaluation are: traditional sort-merge join (Basic), DigestJoin with naive page fetching strategy (Digest(Naive)), DigestJoin with pagebased fetching strategy (Digest(Page)), and DigestJoin with graph-based fetching strategy (Digest(Graph)).

We run all the experiments on a desktop PC equipped with a Core 2 Quad Q6600 CPU and 4GB main memory. The system parameters used in the evaluation are listed in Table 3 . The results of various join algorithms are compared with the $\mathrm{I} / \mathrm{O}$ cost measured in seconds, where the I/O cost is the sum of time used in all kinds of $\mathrm{I} / \mathrm{O}$ operations including sequential

\begin{tabular}{ll}
\hline Parameter & Setting \\
\hline Page size & $4 \mathrm{~KB}$ \\
Ratio of tuple size to & \\
$\quad$ digest entry size $(p)$ & 10 \\
Average tuples per page $(t)$ & 25 \\
Table size & $32 \mathrm{MB}$ (i.e., 8,192 pages) \\
Join selectivity & $0.005 \sim 0.5(0.05$ by default $)$ \\
Memory size & $256 \sim 8,192$ pages (512 by default) \\
\hline
\end{tabular}

reads/writes and random reads/writes.

\section{B. Parameter $k$ of Graph-based Page Fetching Strategy}

In the first set of experiments, we determine the proper configuration of the parameter $k$ for the graph-based page fetching strategy. Recall that $k$ is a parameter to determine the required cache size of a join graph. Fig. 6 shows the I/O results when $k$ is varied from 0.5 to 4 . As can be seen, the value $k$ should not be set smaller than 1 . When it is set at 0.5 , the memory space for caching fetched pages is too small, which leads to frequent page swaps. On the other hand, when the parameter $k$ is increased from 1 to 4 , the $\mathrm{I} / \mathrm{O}$ cost is almost the same. The indicates that for the page cache, it is enough to reserve as many pages as the size of the segment we may select. Reserving more memory only could restrict the join graph not to grow large enough and thus slightly degrade the performance of page fetching. Therefore, we set $k$ at 1 for the graph-based page fetching strategy in the rest evaluation of the DigestJoin method.

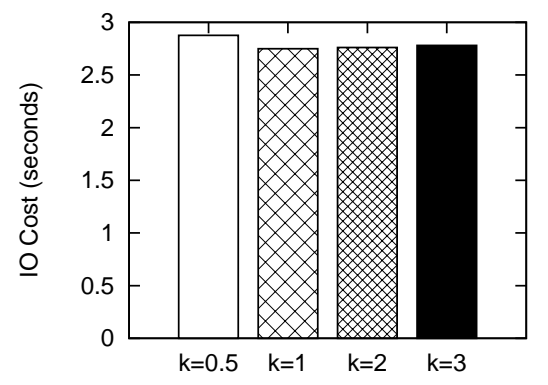

Fig. 6. Impact of Parameter k for Graph-based Fetching Strategy (Selectivity $=0.05$, Memory Size $=512$ Pages, Uniform Join Distribution)

\section{Impact of Join Selectivity}

We now compare different join methods and page fetching strategies, and investigate how the join selectivity would affect their performances. Fig. 7 and Fig. 8 show the comparison results with join selectivity varying from 0 to 0.1 and from 0.1 to 0.5 , respectively. The results do not include the cost to output the final join results, since usually they are not output to the secondary storage and this cost is the same for all join algorithms. As such, the result of Basic remains the same over different selectivity settings. It is used as the baseline in the performance analysis. 
As can be seen from Fig. 7, Digest(Naive) outperforms Basic only when the selectivity is very low (i.e., $<0.08$ ). When the selectivity is higher than 0.08 , as the naive page fetching strategy results in too many duplicate page accesses, it becomes worse than Basic. Similarly, Digest(Page) has the best performance among all join algorithms under a low selectivity, but its performance degrades when the selectivity increases. When the selectivity is higher than 0.3 (see Fig. 8), it performs worse than Basic. Overall, Digest(Graph) offers the best performance and outperforms Basic by $15 \%-64 \%$ for all selectivity setting. As predicted, because of the increased page fetching cost, its performance improvement becomes smaller when the selectivity increases.

\section{Effect of Graph-based Fetching Strategy}

Fig. 9 shows the effect of our graph-based fetching strategy by comparing it with the original heuristic in [3] (for short, Original). Specifically, we implemented Original by allowing it to use as much memory as possible to store the join graph. When the join graph is larger than the memory available, we process some edges by following the heuristic. Since most of memory has already been occupied by the join graph, we have to load the pages on demand. The original heuristic is not expected to be efficient and we can see that, in Fig. 9, after the selectivity is higher than 0.2 , it is not only worse than Digest(Graph), but also even worse than the traditional sort-merge join algorithm (Basic). On the other hand, when the selectivity is small $(<0.2)$, Original is as efficient as Digest(Graph). This is because a low selectivity makes the join graph smaller enough to be held in memory, and since Original and Digest(Graph) follow nearly the same heuristic, their performances are similar.

\section{E. Impact of Page Size}

Fig. 10 and Fig. 11 show the performance comparison with page size varying from $4 \mathrm{~KB}$ to $32 \mathrm{~KB}$. By default, the page size is $4 \mathrm{~KB}$, which is the smallest page size supported by our SSD; and the memory space available for joins is 2 MB (512 pages). When varying the page size, we maintain the same amount of memory (i.e., $2 \mathrm{MB}$ ). Hence, there are 256/128/64 memory pages that could be used when the page size is $8 / 16 / 32 \mathrm{~KB}$, respectively. From Fig. 10 and Fig. 11, the page size has a different impact on the join algorithms. Basic and Digest(Page) become better when the page size increases. The reason is that a big page size implies fewer pages of tables, and the external sort can be done in fewer runs for Basic and Digest(Page). Digest(Graph) also benefits from a big page size. This is because fewer pages of tables result in a smaller join graph, and thus we can make the in-memory join graph more informative, which leads to better page fetching schedules. On the other hand, Digest(Naive) performs worse when the page size increases, and it is even worse than Basic when the page size is bigger than $8 \mathrm{~KB}$ with a low selectivity (see Fig. 10). This is because no matter how few the pages are, the number of fetching requests remains the same under a fixed selectivity.
As such, when the cost of fetching a page become higher due to a bigger page size, Digest(Naive) becomes worse.

\section{F. Impact of Memory Size}

Fig. 12 shows the performance comparison with memory size ranging from 256 to 8192 pages (i.e., $3.1 \%$ to $100 \%$ of the table size). As expected, the I/O cost decreases for all algorithms when more memory space is available. When the memory space is large enough (i.e., 100\%) to hold the entire join table, Basic has the best performance. However, such situation rarely occurs in practice, especially when considering database applications on flash-based mobile devices, which often have very limited memory space.

It can be also observed that when the memory size increases from $3.1 \%$ to $100 \%$ of the table size, the performance gap between Digest(Page) and Digest(Graph) becomes larger (from $6 \%$ to $13 \%$ ). This is because a large memory space is more helpful for the page-based strategy to join the candidate join tables, thereby achieving a more efficient memory usage.

\section{G. Impact of Join Result Distribution}

In the previous experiments, all algorithms are evaluated under the setting where join results are uniformly distributed over the disk pages. In this subsection, we evaluate their performances when the join results follow non-uniform distributions. Specifically, the join results are skewed over the disk pages based on a Zipf distribution. The Zipf distribution is controlled by a skewness parameter of $\theta$. When $\theta$ is 0 , the distribution is uniform. The larger is the value $\theta$, the more skewed is the distribution. We apply the Zipf distribution to one table only, which emulates the case that one table is joined with a part of another table, e.g., some day's orders are joined with the whole customer table.

Fig. 13 shows the results under the Zipf distribution. Due to the skewed distribution of join results, the selectivity cannot be very high. We set it to be 0.1 . We make two observations from Fig. 13. First, when $\theta$ becomes larger, DigestJoin has a better performance improvement over Basic. Furthermore, Digest(Naive) outperforms Basic in most cases. This is because fewer pages are to be fetched when join results have high skewed distributions. Second, from Fig. 13, Digest(Graph) saves more $\mathrm{I} / \mathrm{O}$ cost than Digest(Page) when $\theta$ is larger. This is because in that case the join graph is generally small and hence an effective page fetching schedule is more likely to be achieved.

\section{H. Impact of Flash I/O Characteristics}

In this subsection, we investigate the impact of read/write speeds on the performances of the join algorithms. We use a scaling factor $\lambda$ to simulate the relative speed of read/write operations. Specifically, we set Read $=\overline{\operatorname{Read}} / \lambda$ and Write $=$ $\overline{W r i t e} * \lambda$, where $\overline{\text { Read }}$ and $\overline{W r i t e}$ are the read/write speeds of the Mtron SSD. With $\lambda$ set larger than 1, the read becomes even faster than the write. With $\lambda$ set smaller than 1 , the read might be slower than the write. In the previous performance evaluation, besides the $\mathrm{I} / \mathrm{O}$ time, we also recorded the number 


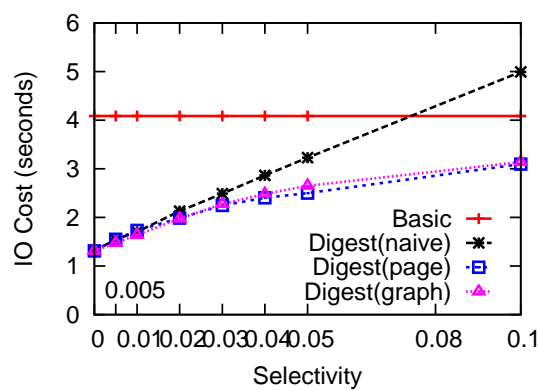

Fig. 7. DigestJoin vs. Traditional Sort-Merge Join under Low Selectivities (Memory Size $=512$ )

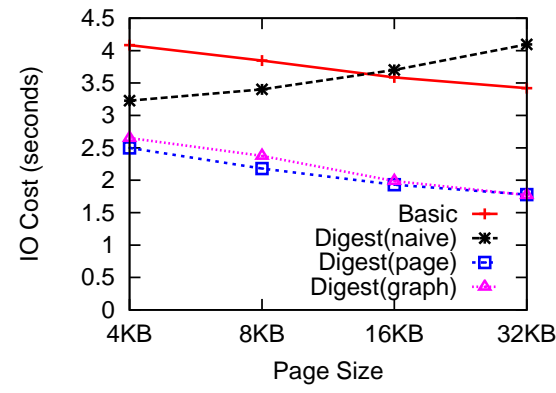

Fig. 10. DigestJoin vs. Sort-Merge Join under Different Page Sizes (Selectivity $=0.05$, Memory Size $=2 M$ )

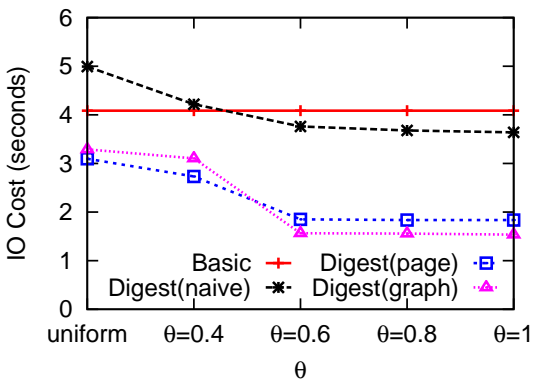

Fig. 13. DigestJoin vs. Sort-Merge Join under Zipf Distribution (Selectivity=0.1, Memory Size=512)

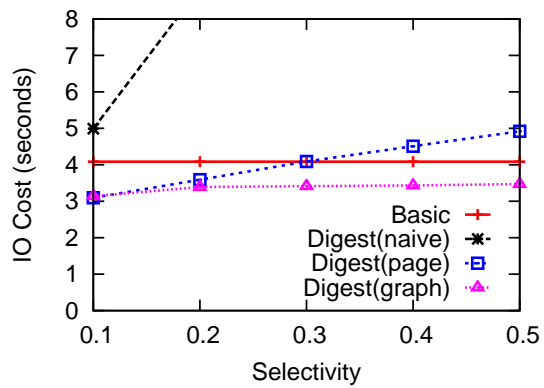

Fig. 8. DigestJoin vs. Traditional Sort-Merge Join under High Selectivities (Memory Size=512)

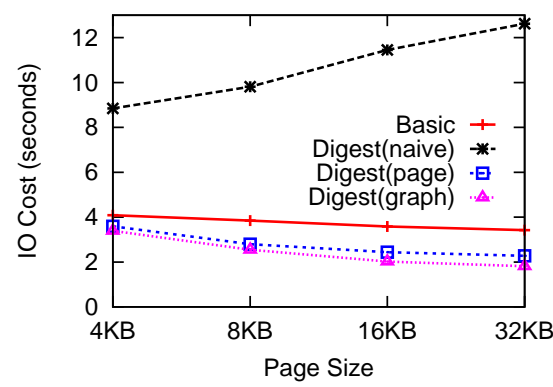

Fig. 11. DigestJoin vs. Sort-Merge Join under Different Page Sizes (Selectivity=0.2, Memory Size $=2 M$ )

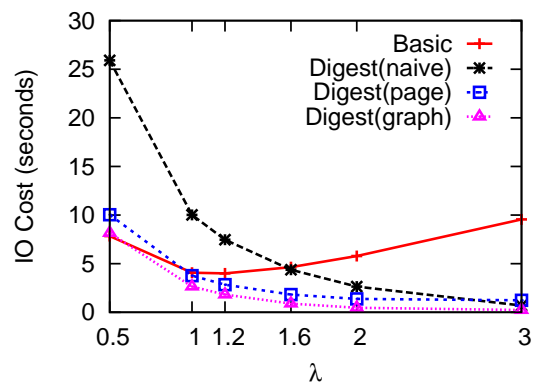

Fig. 14. DigestJoin vs. Sort-Merge Join under Flash I/O Characteristics (Selectivity=0.05, Memory Size $=512$ )

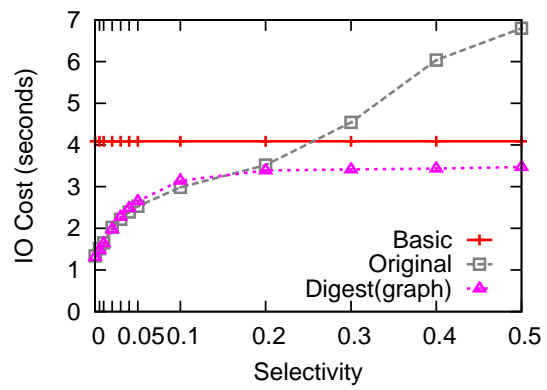

Fig. 9. DigestJoin vs. Original Implementation (Memory Size=512)

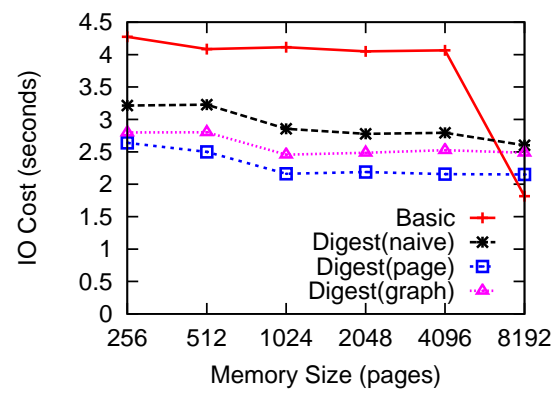

Fig. 12. DigestJoin vs. Traditional Sort-Merge Join under Various Memory Sizes (Selectivity $=0.05$ )

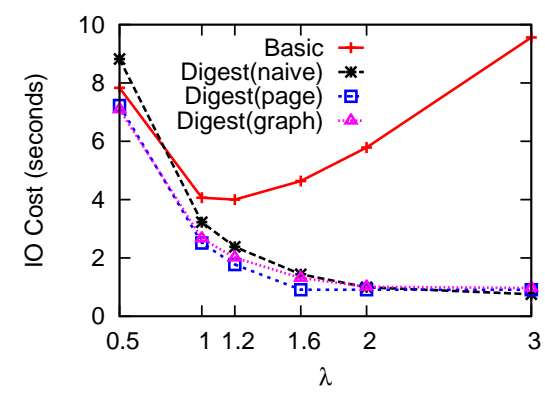

Fig. 15. DigestJoin vs. Sort-Merge Join under Flash I/O Characteristics (Selectivity $=0.2$, Memory Size $=512$ ) of random reads, random writes, sequential reads and sequential writes. Based on such data, we plot the estimated I/O cost under different settings of $\lambda$ in Fig. 14 and Fig. 15, where the selectivity is set at 0.05 and 0.2 , respectively.

We observe that when the relative read/write speed becomes larger (i.e., when $\lambda$ becomes larger), DigestJoin demonstrates a better improvement over Basic. The reason is that with DigestJoin, we reduce the table size to sort and thus save a lot of write operations. When the write cost tends to dominate the overall performance, the advantage of DigestJoin becomes more obvious. Another interesting point is that, with a faster read operation (e.g., $\lambda=3$ ), the performances of different page fetching strategies converge. The reason is that, as page fetching involves read operations only, its cost becomes negligible when the read speed is extremely fast.

\section{RELATED WORK}

Relational database management on flash-based storage media has attracted increasing research attention in recent years. Early work focused on how to assemble flash chips to simulate traditional hard disks [9], [5], [10] and how to extend the lifetime of flash disks [4], [6], [10]. Based on these research efforts, recent work have exploited the characteristics of flash disks to enhance the performance of RDBMSs. In view of the asymmetric read/write speed and the erase-before-write limitation, $\mathrm{Wu}$ et al. [22] proposed a log-based indexing scheme for flash memory. Observing that the log-based indexing scheme is not suitable for readintensive workload on some flash devices, Nath and Kansal [17] developed an adaptive indexing method that adapts to the workload and the underlying storage device. Lee and 
Moon [11] presented a novel storage design called in-page logging (IPL) for RDBMSs. Lee et al. [12] investigated how the performance of standard RDBMS algorithms are affected when the conventional magnetic hard disks are replaced by flash disks. Shah et al. [19] presented a fast scanning and joining method by adapting the PAX storage model [1] to flash disks, which appears the most related work to our study. The main difference, however, is that our work utilizes fast random reads to optimize traditional join algorithms, while PAX presented in [1] is an alternative scheme for storing relations on flash disks.

Join has been one of the important query operators in RDBMSs. Extensive research efforts have been spent on the optimization of join processing. Mihra and Eich [15] surveyed a number of join algorithms and their implementations. In this paper, we focus on exploring the possibility of further improving non-index join algorithms by utilizing fast random reads of flash disks. In particular, our proposed algorithm is inspired by join indexes and page fetching. The idea of join indexes [21], [20], [13] is to precompute join results and record pairs of tuple ids that satisfy the join to speed up future join requests. We find that this idea is useful even when we generate the join indexes on demand for joins on flash disks, i.e., the first phase of DigestJoin. The problem of determining an optimal page fetching schedule in the second phase of DigestJoin is actually found in index join algorithms. Specifically, index join algorithms first compose a list of tuple pairs that participate in the join by using indexes, and then tuples themselves have to be fetched to construct the final results [2], [7]. Merrett et al. [14] proved the decision problem of optimally scheduling the page fetching to be NP-complete. A number of heuristics have been developed, e.g., [8], [18] and [3]. We adapt the ideas behind these heuristics to design page-based and graph-based strategies for page fetching by taking into consideration the memory space constraint.

\section{CONCLUSION}

In this paper, we have proposed a new join method called DigestJoin by exploiting fast random reads of flash disks. DigestJoin is a generic join method as its implementation could invoke any traditional join algorithm. We have identified a critical issue called page fetching for DigestJoin. Three heuristic-based strategies, namely, naive, page-based and graph-based fetching, have been proposed. We have also implemented DigestJoin with these page fetching strategies in an experimental database system on top of a real flash disk. The evaluation results show that DigestJoin generally improves the traditional sort-merge join algorithm under various system parameter settings. In particular, DigestJoin achieves more performance improvement for the scenarios where the read speed is much faster than the write speed. Among those page fetching strategies, the graph-based strategy offers the best overall performance, whereas the page-based strategy wins under a low selectivity and uniform join distribution.

Regarding future work, we plan to extend this study along a few directions. Firstly, we may extend our experiments to analyze both CPU and IO costs of DigestJoin. Secondly, to explore the possibilities of "backward compatibility", we may study the performance of DigestJoin on magnetic disks. Finally, we may also optimize the current implementation of our prototype used in the experiments. These extensions may make DigestJoin more appealing when compared to traditional join algorithms.

\section{ACKNOWLEDGMENT}

The authors are grateful to Prof. Qiong Luo for her constructive comments on an early version of this paper. This work was supported by the Research Grants Council of Hong Kong (Grants HKBU210808 and HKBU211307) and Natural Science Foundation of China (Grant No. 60833005).

\section{REFERENCES}

[1] A. Ailamaki, D. J. DeWitt, M. D. Hill, and M. Skounakis. Weaving relations for cache performance. In $V L D B$ '01, pages 169-180, 2001.

[2] M. W. Blasgen and K. Eswaran. Storage and access in relational databases. IBM System Journal, 16(4):363-377, 1977.

[3] C. Y. Chan and B. C. Ooi. Efficient scheduling of page access in index-based join processing. IEEE Trans. on Knowl. and Data Eng., 9(6):1005-1011, 1997.

[4] L. Chang. On efficient wear leveling for large-scale flash-memory storage systems. In SAC'07, pages 1126-1130, 2007.

[5] L. Chang, T. Kuo, and S. Lo. Real-time garbage collection for flashmemory storage systems of real-time embedded systems. Trans. on Embedded Computing Sys., 3(4):837-863, 2004.

[6] Y. Chang, J. Hsieh, and T. Kuo. Endurance enhancement of flashmemory storage systems: an efficient static wear leveling design. In $D A C^{\prime} 07$, pages 212-217, 2007.

[7] J. M. Cheng, D. J. Haderle, R. Hedges, B. R. Iyer, T. Messinger, C. Mohan, and Y. Wang. An efficient hybrid join algorithm: A db2 prototype. In ICDE, pages 171-180, 1991.

[8] F. Fotouhi and S. Pramanik. Optimal secondary storage access sequence for performing relational join. IEEE Trans. on Knowl. and Data Eng., 1(3):318-328, 1989.

[9] A. Kawaguchi, S. Nishioka, and H. Motoda. A flash-memory based file system. In USENIX Winter, pages 155-164, 1995.

[10] H. Kim and S. Lee. A new flash memory management for flash storage system. In COMPSAC'99, page 284, 1999.

[11] S. Lee and B. Moon. Design of flash-based dbms: an in-page logging approach. In SIGMOD '07, pages 55-66, 2007.

[12] S.-W. Lee, B. Moon, C. Park, J.-M. Kim, and S.-W. Kim. A case for flash memory ssd in enterprise database applications. In SIGMOD, pages 1075-1086, 2008.

[13] Z. Li and K. A. Ross. Fast joins using join indices. The VLDB Journal, 8:1-24, 1999.

[14] T. H. Merrett, Y. Kambayashi, and H. Yasuura. Scheduling of pagefetches in join operations. In $V L D B$ '81, pages 488-498, 1981.

[15] P. Mishra and M. H. Eich. Join processing in relational databases. ACM Comput. Surv., 24(1):63-113, 1992.

[16] D. Myers. On the use of nand flash memory in hgh-performance relational databases. Master's thesis, MIT CSAIL, Cambridge, MA, December 2007.

[17] S. Nath and A. Kansal. Flashdb: Dynamic self-tuning database for nand flash. Technical Report MSR-TR-2006-168, Microsoft Research, 2006.

[18] E. R. Omiecinski. Heuristics for join processing using nonclustered indexes. IEEE Trans. Softw. Eng., 15(1):18-25, 1989.

[19] M. A. Shah, S. Harizopoulos, J. L. Wiener, and G. Graefe. Fast scans and joins using flash drives. In DaMoN08, pages 17-24, 2008.

[20] P. Valduriez. Optimization of complex database queries using join indices. Database Engineering, 9(16):10-16, Dec. 1986.

[21] P. Valduriez. Join indices. ACM Trans. Database Syst., 12(2):218-246, 1987.

[22] C. Wu, T. Kuo, and L. P. Chang. An efficient b-tree layer implementation for flash-memory storage systems. Trans. on Embedded Computing Sys., 6(3):19, 2007. 